\title{
IMPACTO DE LOS COSTOS DE SERVICIOS DE FABRICACIÓN EN LAS ESTRUCTURAS DE COSTOS - ANÁLISIS DE CONTRATOS
}

\section{IMPACT OF SERVICE COSTS IN MANUFACTURING COST STRUCTURES - ANALYSIS OF CONTRACTS}

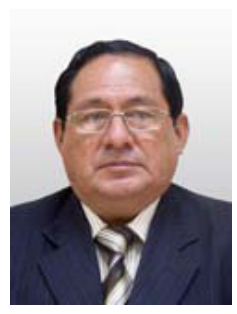

Agustin Rodriguez Chavez *

Docente Asociado de la Facultad de Ciencias Contables - UNMSM

Colaboradora: Ingrid Rodriguez Castro

[Recepción: Agosto de 2011/ Conformidad: Octubre de 2011]

\section{RESUMEN}

Con nuestro artículo ESTRUCTURAS FLEXIBLES DE COSTOS PARA ENFRENTAR LA GLOBALIZACION Y LA COMPETITIVIDAD EN LAS EMPRESAS, presentado en la revista QUIPUKAMAYOC $\mathrm{N}^{\circ} 33$ iniciamos una investigación orientada a proponer un modelo que recoja la cada vez más utilizada estrategia para lograr competitividad de costos en el mercado.

Las empresas, ante el nuevo modelo de competitividad que exige flexibilizar los procesos de producción, están implementando estrategias empresariales con la finalidad de ACELERAR, SIMPLIFICAR y ASEGURAR procesos.

Las empresas de servicios son parte de esta estrategia, a las cuales se les traslada funciones o actividades que se realizaban dentro de las empresas. Pero tal como expusimos en dicho artículo, los costos de la TERCERIZACION o SERVICIOS ya no puede tratarse dentro de los otros costos indirectos de producción (CIP) por lo que estamos en BUSCA DEL CUARTO ELEMENTO, como propuesta para administrar los costos con mayor precisión. En esta oportunidad, analizaremos los diversos contratos de servicios para comprender porque los costos asociados a dichas estrategias deben ser ACUMULADOS, MEDIDOS y CONTROLADOS en forma separada.

Palabras Clave: Contratos de servicios, procesos productivos, procesos de negocio, tercerización, estructura de costos.

\begin{abstract}
ABSTRAC
With our article FLEXIBLE COST STRUCTURES TO OVERCOME THE GLOBALIZATION AND COMPETITIVENESS IN THE COMPANIES, presented in the journal QUIPUKAMAYOC No 33 we began an investigation to propose a model that incorporates the increasingly used strategy to achieve cost competitiveness in the market.

Companies, with the new model of competitiveness that demands flexible production processes, are implementing business strategies in order to SPEED UP, SIMPLYFY and SECURE processes.

Service companies are part of this strategy, which they are transferred functions or activities carried out within companies. But as we discussed in this article, SERVICE or OUTSOURCING costs can no longer be treated inside the other indirect costs of production (ICP) so we are looking for the fourth element, as a proposal to manage costs more accurately.

This time, we will analyze various service contracts to understand because the costs associated with such strategies should be ACUMULATED, MEASURED and CONTROLLED as separately.
\end{abstract}

Key words: Service contracts, production processes, business processes, outsourcing, cost structure

* Contador Publico Colegiado Certificado, Maestria en Costos y Presupuestos, Doctorando en Ciencias Empresariales. Especialista en Costos. E-mail: arodriguezc@unmsm.edu,pe. Colaboradora Ingrid Rodiguez Castro alumna de Pregrado de La Facultad de Ciencias Contales, UNMSM. 


\section{INTRODUCCION:}

El mundo está en una búsqueda incesante de transformarse, originando que los hábitos y costumbres para la adquisición de bienes y servicios obliguen a que los procesos de negocios se adecuen a estas transformaciones, como sucede con los mercados y comunidades a través de redes sociales. Por ejemplo, los países en general hacen esfuerzos de cambios en sus estructuras, probablemente obligados, por el fenómeno de la globalización creando accesos y eliminando barreras a las nuevas tecnologías, ampliando las concesiones de uso a los accesos, a las tecnologías como comunicaciones.

Las empresas frente al riesgo de invertir en tecnologías que pueden ser recuperadas en un período definido, quedándose obsoletos y perdiendo competitividad o por limitaciones en los accesos a fuentes de financiamiento, buscan mantener su competitividad a través de tercerizaciones para la adquisición de bienes y servicios.

Ivancevich en su libro "Gestión, calidad y competitividad (1996)" cita la siguiente definición de competitividad: "Competitividad Nacional, la medida en que una nación, bajo condiciones de mercado libre y leal es capaz de producir bienes y servicios que puedan superar con éxito la prueba de los mercados internacionales, manteniendo y aún aumentando al mismo tiempo la renta real de sus ciudadanos".

Esta definición puede adaptarse al ámbito organizacional, considerando como competitividad, la medida en que una organización es capaz de producir bienes y servicios de calidad, que logren éxito y aceptación en el mercado global.

La tercerización para la adquisición de bienes intermedios, semi elaborados o componentes se convierte finalmente en un elemento del costo como material directo obligando a las empresas a tener un buen planeamiento y gestión de inventarios.

Por la tercerización como servicio, de un proceso productivo, poco se ha escrito y menos sobre su impacto en las estructuras de costos originadas por las diversas modalidades de contratos. Más aún, de lo que expresamos en el artículo anterior, existe confusión entre producción de bienes intermedios y servicios de fabricación, así como recogimos precisiones que la norma tributaria ha desarrollado para la aplicación de las detracciones. Los contratos buscan desarrollar en las empresas procesos de producción flexibles para mejorar la competitividad, acelerando el tiempo de respuesta a las demandas de un mercado global. Hoy muchos negocios y/o productos pierden por llegar tarde al mercado.

Por otro lado, la tercerización de los procesos a través de contratos de servicios desarrolla integraciones horizontales, incluyendo en ellas clausulas de compromisos, de alianzas estratégicas para ASEGURAR, ACELERAR Y SIMPLIFICAR PROCESOS basados en la teoría de cadena de valor extendida de Porter.

La tercerización de las organizaciones está centrada en el sistema japonés. Este modelo se basa «en una política de fuerte tercerización con los proveedores; alude a relaciones de transferencia hacia otras empresas de etapas y tercerización del proceso» (Novick, 1999:18). Lo que pareciera la existencia de un convenio entre un conjunto de firmas, marcas para compartir riesgos y auxiliarse en el desarrollo productivo fuera de la organización matriz.

En el actual contexto, el Outsourcing surge como una atractiva herramienta de gestión de negocios que procura la maximización de beneficios y la minimización de costos, a la vez que genera valor para la empresa y sus grupos de interés. 
Las empresas de servicios son parte de la estrategia de tercerización, que en su mayoría está conformada por empresas pequeñas o medianas que cumplen funciones o actividades fuera de la empresa contratante o en el mismo espacio de trabajo. Este tipo de tercerización implica ciertas exigencias de acuerdo a las calificaciones o competencias que requiera cumplir cada eslabón del proceso productivo.

Ahora bien, en esta perspectiva el tratamiento del servicio que se brinda es otorgado por las empresas de acuerdo a modalidades de contratación de servicios de fabricación que son de mutuo acuerdo con los clientes, con el fin de abrir nuevos mercados y poder posteriormente, de acuerdo al tipo de contrato de fabricación, hacer los costos de las empresas.

Ante este contexto, este trabajo tiene como propósito analizar como las modalidades de contratos de servicio de fabricación en las empresas con el fin de ser competitivos en el mercado globalizado impacta en las estructuras de costos.

La metodología utilizada en esta investigación consistió en una revisión teórica del fenómeno de la tercerización, lo que permitió caracterizar los modelos de contratos, reiterar la importancia del entorno global el cual es tan competitivo y lo más importante, identificar las variables para luego evaluar su comportamiento e impacto en las estructuras de costos.

\section{FACTORES DE COMPETITIVIDAD DEL SERVICIO DE FABRICACION:}

i. Simplificación de procesos: La competitividad se puede demostrar por la labor que se realiza constantemente a través de la utilización de planes organizados, que sirven para la aplicación de mejores técnicas que faciliten la ejecución de las tareas; es decir, que ayude a disminuir o reducir la cantidad de esfuerzo requerido para ejecutar una labor determinada $y$ que es concebida fundamentalmente como una tarea permanente con el objeto de minimizar las tareas ordinarias propias de toda empresa. La tendencia a la automatización de procesos de tejido, remallado, envasado en una fábrica de medias, hoy se efectúan en una sola máquina pero requiere de grandes volúmenes para su adquisición; por lo que, las empresas prefieren tomar el servicio.

ii. Aceleración de procesos: Se destaca que la aplicación del Outsourcing en el sector empresarial permite acelerarla percepción de los beneficios obtenidos del rediseño radical de los procesos, empleando herramientas de Reingeniería, logrando que empresas ajenas a nuestro quehacer diario hayan experimentado una revisión fundamental de sus procesos, con mejoras significativas en rendimiento como el costo de la calidad, el servicio y la velocidad de respuesta. Los servicios de horneado de panetones en un horno continuo hoy acelera el proceso frente a hornos por lotes, pero su utilización requiere de volúmenes mínimos para su utilización.

iii. Aseguramiento de procesos: Se brinda un aseguramiento del proceso mediante el Outsourcing cuando se realiza actividades que permiten a las empresas acceder a la posibilidad de implantar dentro de sus procesos, actividades de mejora que eventualmente reditúan en una mejor calidad de sus productos y/o servicios, así como también la reingeniería, para lograr mejoras sustantivas en medidas de desempeño. Hoy existen empresas que brindan servicios que garantizan la calidad del envasado de acuerdo a las exigencias de presentación de los mercados como el de Japón. 


\section{GESTIÓN DE COSTOS DE SERVICIOS}

La alianza estratégica entre la empresa y sus Outsourcer pasa por que la primera pueda lograr una buena gestión de competitividad a costos razonables, desarrollando constantes actividades de análisis entre hacer sus procesos o comprar servicios. Para esto es necesario tener claro la medición, control y acumulación de los servicios y sus costos asociados, veamos que entendemos por ello.

i. Medición de procesos: Para realizar una adecuada medición del servicio es necesario establecer con claridad los tiempos y volúmenes para cada uno de los procesos, actividades y componentes contratados, especialmente en lo relacionado al material entregado a utilizar. La determinación de las mermas y reprocesos afectan a los costos de los servicios en diversas formas de acuerdo a la modalidad del servicio. En la industria textil es un práctica que el Outsourcer deba comprar las mermas adicionales a lo establecido.

ii. Control de procesos: Conociendo como se obtiene cierta cantidad de producción de trabajo, se puede mejorar los métodos empleados para su realización, reajustar la asignación de tareas, aliviar en general la carga de trabajo y eliminar congestiones. Los clientes de acuerdo a la modalidad del servicio destacan usualmente personal de ingeniería para el planeamiento y control de la producción en el Outsourcer.

iii. Acumulación de costos: Un sistema de acumulación es apropiado cuando un producto o lotes de productos se manufacturan de acuerdo a las especificaciones de un cliente, y el valor del servicio es identificado con claridad al producto; sin embargo, algunos contratos en los que se incluye el uso de una capacidad de volumen disponible, cuando no se utilizó la capacidad pactada, no es posible asignar los costos directamente a los productos.

\section{ANTECEDENTES DE LOS CONTRATOS DE OUTSOURCING - TERCERIZACIÓN}

De acuerdo a lo expuesto por Daniel Echiz, se puede mencionar que los contratos de Outsourcing tienen sus antecedentes según las dos siguientes posiciones que explican su nacimiento:

i. Por un lado, es la posición más aceptada aquella que explica que el Outsourcing nace incipientemente en los años setenta del siglo pasado con la revolución post-industrial, y encuentra su punto de ebullición dos décadas después con la revolución informática, para culminar con el modelo inspirado en la industria del automóvil Ford. Durante este período, la empresa estadounidense se convierte en gran empresa, dedicándose no sólo a su negocio principal, sino también a actividades satelitales que, aunque no son esenciales, sí son necesarias. Los modelos evolucionaron hacia sistemas más flexibles que permitieron la tercerización de procesos especialmente si la organización logra una gestión a partir de la identificación de la cadena de valor de sus productos y logra lazos fuertes entre sus proveedores de servicios y clientes.

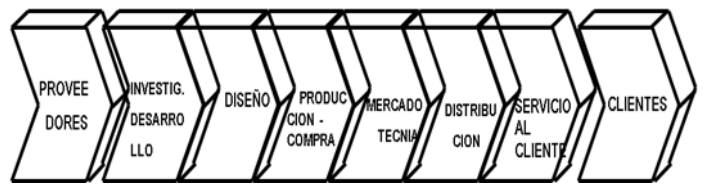


ii. Por otro lado, se encuentra la posición que sostiene que en Italia la economía informal es la creadora de la especialización flexible, antecedente del Outsourcing; en efecto, surgió a propósito de la informalidad del sector agrario en su paso a la economía agroindustrial, donde la pequeña empresa agraria necesitaba de servicios especializados del sector informal (por tener menor costo de servicios) y de donde nace la entonces incipiente figura del Outsourcing.

iii. De estas dos tesis que explican la aparición del Outsourcing: la economía de gran escala y la economía informal; sin embargo, la primera de ellas la que ha recibido mayor aceptación por parte de la doctrina.

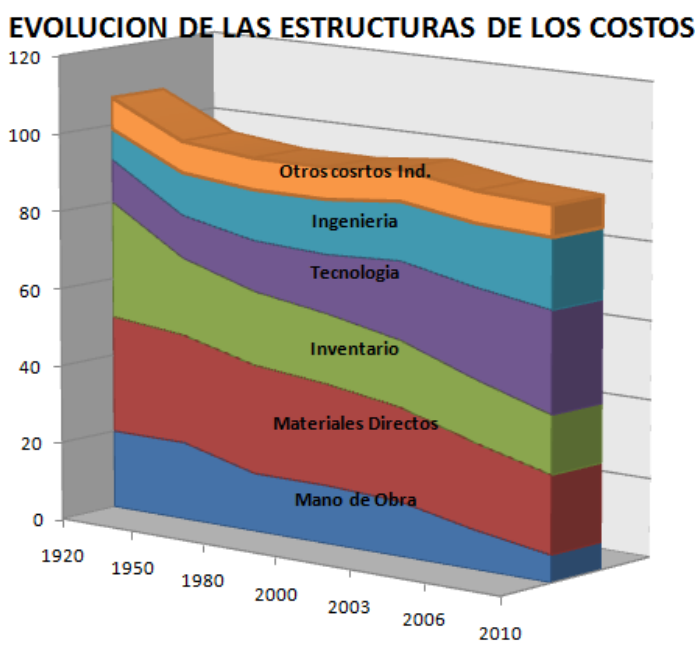

CONTRATO DE OUTSOURCING

El contrato de Outsourcing es un modelo de contrato mediante el cual se regula la tercerización de servicios. Por tanto, es un contrato de tercerización de determinados servicios u obras (también denominado "contrato de externalización”, pero es más conocido en su terminología inglesa, "Outsourcing") que consiste en la realización por parte de una empresa o profesional de los trabajos y funciones encomendados por la empresa que contrata. i. Partes del Contrato: En el contrato de Outsourcing intervienen dos partes contratantes:

i.i. Por un lado, la empresa que contrata el Outsourcing: En este caso, nos adscribimos a la denominación que le otorga la doctrina uruguaya, empresa cliente que, en términos generales, es la que decide cuál es la actividad que se delegará a la empresa de Outsourcing.

i.ii. Por otro lado, el Outsourcer: A quién se le delega el proceso, esto es, la tarea de realizar la actividad de la empresa cliente, porque cuenta con el know-how del negocio.

ii. Contenido de un contrato de servicios: El contrato de Outsourcing debe definir algunos de los siguientes aspectos:

- Su duración.

- Las condiciones de la cesión de los activos (tanto económicas como de otro tipo) referidos al momento inicial del acuerdo entre el Outsourcing y Outsourcer.

- Las condiciones de la gestión de los SI a llevar a cabo durante el contrato (nivel de servicio).

- Las condiciones de recuperación de la gestión de los SI una vez finalizado el contrato.

- La propiedad intelectual, especialmente si se traspasa al proveedor la responsabilidad del desarrollo de aplicaciones.

- Las condiciones previstas para la resolución del contrato con anterioridad a la fecha de su finalización prevista.

iii. Modelo de Contrato de Servicio de Fabricación:

\section{CONTRATO DE PRESTACION DE SERVICIOS}

Conste por el presente documento, el contrato de servicios para el diseño y elaboración de un modelo de costeo aplicable al sistema 
de costos a implementar; que celebran ... en los términos y condiciones siguientes:

- PRIMERA CLAUSULA.- ANTECEDENTES:

ECONSULTING, ha presentado una propuesta para el servicio de fabricación; propuesta que ha sido aceptada por LURIN en las condiciones que se detallan en las cláusulas siguientes.

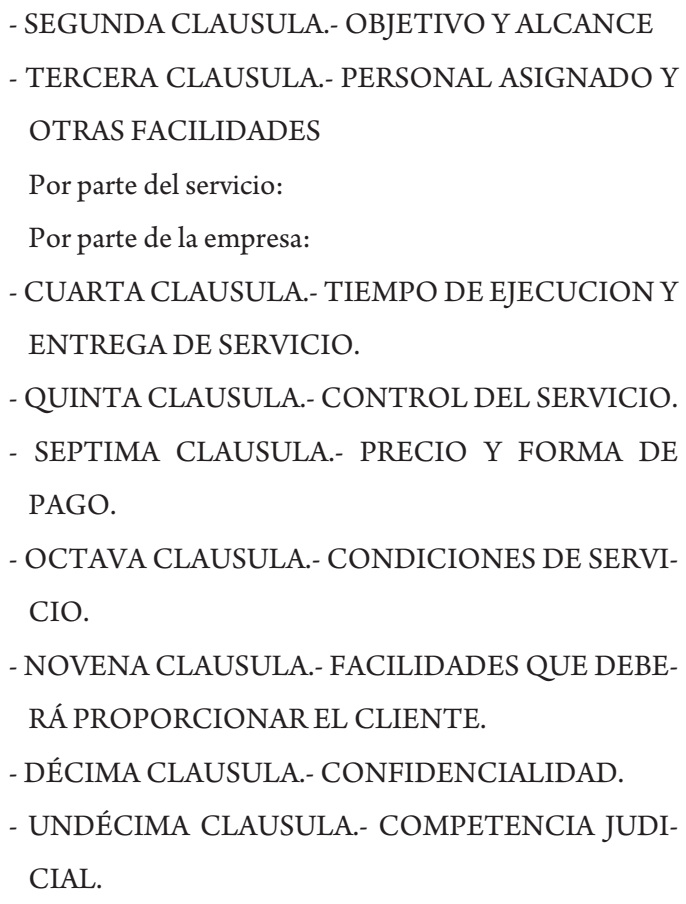

\section{CLASES DE CONTRATOS DE SERVICIOS DE FABRICACIÓN:}

\section{i. Servicios por Volumen - Productos:}

Este modelo de contrato se aplica en los servicios de fabricación de grandes volúmenes de productos, entre otras, con el fin de obtener el producto terminado, semi elaborado o componentes de un producto. Responde a las estrategias de simplificación y aceleramiento de procesos. En este caso, los contratos incluyen con claridad la unidad que servicio para el pago del servicio; por ejemplo, en la industria textil el servicio de teñido de telas o prendas se cobra por kilos. ii. Servicios por Horas: Este modelo de contrato se aplica en los servicios de fabricación de productos en donde es importante el uso de mano de obra y/o maquinaria especializada sin tener volumen de producción. Responde a estrategias de aceleración y aseguramiento de procesos. Estos tienen en cuenta un tiempo estándar de fabricación de referencia para determinar el costo del servicio brindado, como el caso del fileteo y cocinado de pescado para conservas.

iii. Servicios por Capacidad de Planta: Este modelo de contrato parte de conocer la capacidad (productos/año) máxima que cada una de las operaciones, puestos manuales y máquinas de producción o transporte normalmente puede producir. La capacidad de operación más baja será la capacidad de la planta. La empresa que brinda el servicio de Outsourcing determina el costo de su servicio teniendo en cuenta que la fabricación de los productos que realice, responde a estrategias de aseguramiento y aceleración de procesos.

iv. Servicios por Órdenes de Trabajo o Lote: Servicio por un producto o lote de producción concreta; por ejemplo, la fabricación de un transformador, por lo que la vida del contrato irá ligada al tiempo que se tarde en realizar dicha obra. Este trabajo se puede realizar dentro de la mima empresa empleadora o fuera de ella. Responde a estrategias de aceleración y aseguramiento de procesos. Las actividades y recursos se gestionan como un todo. Su coordinación adquiere carácter crítico. Existe un connotado interés por el control de los costos y las fechas de terminación el servicio se paga por la orden. 
v. Contrato Eventual por Circunstancias de la Producción: Se utiliza normalmente en los períodos o temporadas en que aumenta la demanda de la empresa como los pedidos de exportación, campañas estacionales y es contratado en combinación de los modelos anteriores.

Es el contrato que más se adapta a las circunstancias especiales del mercado: la función del contratado será la de reforzar la plantilla cuando las prisas lo requieran.

vi. La Maquila o lo que hoy se denomina los Clúster: Este tipo de relación, de acuerdo a Gómez (2003), constituye esencialmente la fase de ensamblado de la cadena productiva de una empresa trasnacional. Se le caracteriza como producción no integrada, en el sentido de que no integra en sí misma las diversas fases de un proceso que acaba en la elaboración de un producto, sino que más bien constituye una de esas fases.

vii. Servicios de Producción incluyendo la Ingeniería de Procesos: Luego de afianzar una alianza estratégica por un periodo que por lo general es el tercer ciclo operativo (DISEÑAR, CONTRATAR, PROCESAR, FACTURAR, COBRAR Y PAGAR), el equipo y procesos están organizados en una línea o líneas especializadas para producir un pequeño número de productos, semi elaborados o componentes diferentes o familias de productos. Estos sistemas se usan cuando el diseño del producto es estable y el volumen es lo suficientemente elevado para hacer un uso eficiente de una línea especializada con capacidades dedicadas. El control del ciclo productivo está automatizado, existe alta estandarización y una elevada eficiencia en todo el proceso.
Existe una diversidad de modelos de servicios que no lo hemos considerado puesto que para el propósito de nuestra investigación y propuesta con lo presentado se nos permite analizar el comportamiento, la clasificación asignación y/o distribución de los costos de los servicios, medir y evaluar el impacto en la estructura de costos. Además, en muchos casos son variantes o combinaciones de los modelos explicados.

Sin embargo, al final del artículo presentamos una matriz para que incluya el modelo de su empresa o el que tenga conocimiento.

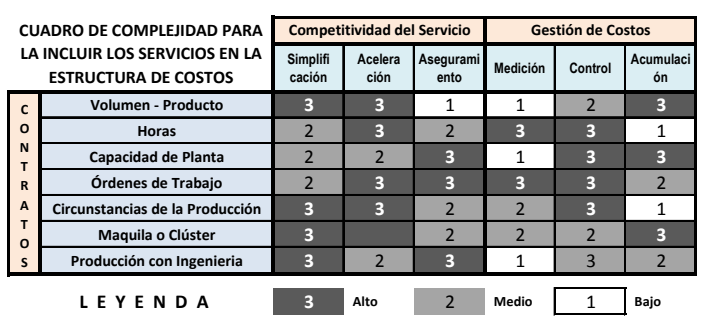

\section{COMPORTAMIENTO DE LOS COSTOS}

Cuando una empresa elige la opción de recibir un servicio de un tercero para incluirlo en su proceso productivo empieza la búsqueda de una minimización de costos de producción. En este caso existe una evaluación comparativa entre el costo de hacer y costo de comprar.

Esta evaluación debe incluir los costos indirectos asociados tales como gestión de personal. Se reducen costos de personal: el valor monetario o costo de la planilla en las empresas ya no forma parte de la empresa contratante del servicio de Outsourcing.

Además dependiendo del modelo de contrato puede convertir los costos fijos en costos variables. Por ejemplo, no se necesita de un ambiente para el taller si es que la empresa contratada realiza la producción fuera del ambiente de la empresa que la contrata. 
Por otro lado, se debe revisar los costos que tradicionalmente se asignaron o distribuyeron a procesos como los de supervisión, almacén, gerencia y otros que con la decisión de contratar no desaparecen. Veamos, por ejemplo, el PROCESO DE ARENADO en la industria metalmecánica donde con un buen contrato de servicio se puede lograr ahorros de hasta $9.09 \%$.

\begin{tabular}{|c|c|c|}
\hline \multicolumn{3}{|c|}{ ESTRUCTURA DE COSTO DE PRODUCIR EN LA EMPRESA } \\
\hline COSTO TOTAL DEL PROCESO & 2010 & $\%$ \\
\hline MATERIALES DIRECTOS & 5,000 & $45 \%$ \\
\hline MANO DE OBRA & 2,000 & $18 \%$ \\
\hline SERVICIOS & 0 & $0 \%$ \\
\hline COSTOS INDIREC.D PROD & 4,000 & $36 \%$ \\
\hline TOTAL & 11,000 & $100 \%$ \\
\hline COSTO DEL SERVICIO & 4000 & $100 \%$ \\
\hline COSTO DEL PROCESO CON SE & & \\
\hline COSTO TOTAL DEL PROCESO & 2010 & $\%$ \\
\hline MATERIALES DIRECTOS & 5,000 & $50 \%$ \\
\hline MANO DE OBRA & 0 & $0 \%$ \\
\hline SERVICIOS & 4,400 & $44 \%$ \\
\hline COSTOS INDIREC.D PROD & 600 & $6 \%$ \\
\hline TOTAL & 10,000 & $100 \%$ \\
\hline REDUCCION DE COSTOS & 1,000 & $9.09 \%$ \\
\hline
\end{tabular}

Cuando la empresa evalúa las limitaciones de inversión en tecnología de procesos sea por barreras en el financiamiento o volumen de producción que no justifica la inversión, al analizar los costos de HACER o COMPRAR la contratación de un servicio de Outsourcing va a permitir una mejor optimización de la calidad, mejora de rendimientos y reducción de costos.

Las empresas que contratan ya no necesitan de la compra de máquinas (si el servicio incluye maquinaria) para la producción.

El Outsourcing modifica los conceptos contables relacionados con la gestión. Se transforman los costos del recurso en costos del servicio; los costos fijos, en costos variables y las inversiones, en gastos desapareciendo los activos.
Por ejemplo, un servicio de desarrollo y corte para la confección de prendas mejora el rendimiento de materiales como la tela, si el servicio incluye el uso de tecnología automatizada de desarrollo al que la empresa no tiene acceso. Así podemos ver que la empresa logra un ahorro del $5.45 \%$ cuando contrata el servicio.

\begin{tabular}{|l|r|r|}
\hline \multicolumn{3}{|l|}{ ESTRUCTURA DE COSTO DE PRODUCIR EN LA EMPRESA } \\
\hline COSTO TOTAL DEL PROCESO & $\mathbf{2 0 1 0}$ & $\%$ \\
\hline MATERIALES DIRECTOS & 8,000 & $73 \%$ \\
\hline MANO DE OBRA & 1,000 & $9 \%$ \\
\hline SERVICIOS & 0 & $0 \%$ \\
\hline COSTOS INDIREC.D PROD & 2,000 & $18 \%$ \\
\hline TOTAL & $\mathbf{1 1 , 0 0 0}$ & $100 \%$ \\
\hline
\end{tabular}

\begin{tabular}{|l|r|r|}
\hline COSTO DEL SERVICIO & $\mathbf{4 , 4 0 0}$ & $100 \%$ \\
\hline
\end{tabular}

\begin{tabular}{|l|r|r|}
\hline COSTO DEL PROCESO CON SERVICIO \\
\hline COSTO TOTAL DEL PROCESO & $\mathbf{2 0 1 0}$ & $\%$ \\
\hline MATERIALES DIRECTOS & 7,500 & $72 \%$ \\
\hline MANO DE OBRA & 0 & $0 \%$ \\
\hline SERVICIOS & 2,500 & $24 \%$ \\
\hline COSTOS INDIREC.D PROD & 400 & $4 \%$ \\
\hline TOTAL & 10,400 & $100 \%$ \\
\hline \begin{tabular}{|l|r|r|}
\hline REDUCCION DE COSTOS & 600 & $5.45 \%$ \\
\hline
\end{tabular}
\end{tabular}

Estos análisis no son posibles cuando la empresa no puede medir y acumular los costos de un proceso o cuando al recibir el servicio es tratado como dentro del elemento de costos indirectos de producción (CIP) dentro de la estructura tradicional de los TRES ELEMENTOS DE COSTOS.

\section{CONCLUSIONES}

i. El reordenamiento de las empresas ha impulsado la tercerización de diferentes secciones $o$ actividades de las organizaciones, así como los procesos productivos, ubicados o repartidos en distintas áreas geográficas que reúnen las condiciones elementales para implantarse en el mercado internacional. Así el desarro- 
llo de los CLUSTER permite el mayor acceso y facilidad de servicios de producción.

ii. La tendencia de las empresas es sustituir las estructuras rígidas y burocráticas por organizaciones planas, descentralizadas y tercerizadas, como una forma de adaptarse a los cambios e incertidumbre del mercado global y competir, lo que impone tomar decisiones para flexibilizar sus organizaciones.

iii. Las modalidades de contratos que se celebran entre las personas u empresas son de carácter muy importante para verificar el desempeño realizado por la parte contratada para cumplir con su fin y lograr una apropiada identificación clasificación y medición de costos asociados al servicio.

iv. El conocimiento del procedimiento y el diseño del servicio de fabricación a desarrollar permite asociar diversos costos involucrados en un determinado centro de costos, y evaluar el resultado de mejoras en los costos de hacer o comprar servicios de producción.

v. Como se observa, las empresas de servicio contribuyen con la tercerización emprendida por las empresas para su expansión y disminución de costos.

vi. Como se puede apreciar, la diversidad de modelos existentes de servicios de producción o tercerización de procesos presiona a las empresas a modificar sus estructuras de costos para una mejor medición y acumulación de costos por lo que nuestra propuesta es la de insertar a la estructura EL CUARTO ELEMENTO.

\section{EN BUSCA DEL CUARTO ELEMENTO}

vii. En la publicación siguiente, analizaremos la medición y acumulación de cos- tos de servicios en una estructura flexible incluyendo modelos de clasificación identificación, asignación y distribución de acuerdo a los modelos estudiados.

viii. En las siguientes publicaciones, incluiremos modelos de medición para evaluar HACER O CONTRATAR SERVICIOS de procesos productivos y mejoras de costos de los procesos tercerizados.

ix. Finalmente y luego de enriquecer la investigación, elevaremos propuesta de modificación en la normatividad tributaria sobre costos y plantearemos una propuesta de modificación de las Normas Internacionales de Contabilidad sobre estos cambios en la estructura de costos.

x. Para el enriquecimiento de esta investigación y los resultados en las investigaciones siguientes necesita un apoyo crítico y autocrítico para lo cual incluyo una matriz para evaluar la complejidad y la gestión de costos de un proceso que se requiera medir:
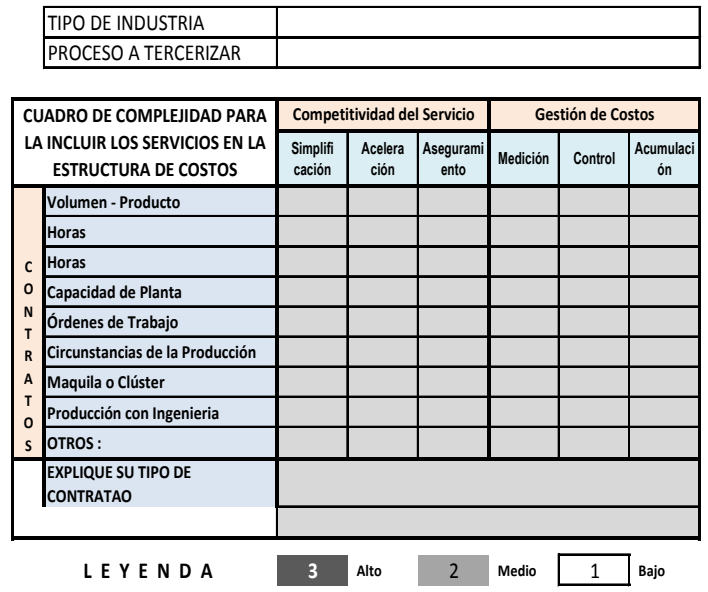

xi. También se incluye una tabla para la identificación de las probables reducciones de costos que espera, teniendo en cuenta el factor de competitividad y gestión de costos más alto identificado. 


\begin{tabular}{|c|c|c|}
\hline \multicolumn{3}{|l|}{ TIPO DE INDUSTRIA: } \\
\hline \multicolumn{3}{|l|}{ TIPO DE PROCESO: } \\
\hline \multicolumn{3}{|l|}{ F. COMPETITIVIDAD: } \\
\hline \multicolumn{3}{|l|}{ GESTION DE COSTOS: } \\
\hline \multicolumn{3}{|c|}{ ESTRUCTURA DE COSTO DE PRODUCIR EN LA EMPRESA } \\
\hline COSTO TOTAL DEL PROCESO & s/ & $\%$ \\
\hline \multicolumn{3}{|l|}{ MATERIALES DIRECTOS } \\
\hline \multicolumn{3}{|l|}{ MANO DE OBRA } \\
\hline \multicolumn{3}{|l|}{ SERVICIOS } \\
\hline \multicolumn{3}{|l|}{ COSTOS INDIREC.D PROD } \\
\hline \multicolumn{3}{|l|}{ TOTAL } \\
\hline \multicolumn{3}{|l|}{ COSTO DEL SERVICIO } \\
\hline \multicolumn{3}{|c|}{ COSTO DEL PROCESO CON SERVICIO } \\
\hline COSTO TOTAL DEL PROCESO & 2010 & $\%$ \\
\hline \multicolumn{3}{|l|}{ MATERIALES DIRECTOS } \\
\hline \multicolumn{3}{|l|}{ MANO DE OBRA } \\
\hline \multicolumn{3}{|l|}{ SERVICIOS } \\
\hline \multicolumn{3}{|l|}{ COSTOS INDIREC.D PROD } \\
\hline TOTAL & & \\
\hline DUCCION DE COSTOS & & \\
\hline
\end{tabular}

\section{REFERENCIAS BIBLIOGRÁFICAS}

1. Charles HORNGREN, CHARLES T, Edición (2006). Contabilidad de costos un Enfoque Gerencial. 12va Ed., México D.F., México.

2. Añez Hernández Carmen (2007). Estrategias de Contratación Añez Hernández Carmen (2007). Estrategias de Contratación.
3. Daniel Echaiz Moreno (2008). El Contrato de Outsourcing. Boletín Mexicano de derecho comparado, nueva serie, año XLI No 122 May - Agos 2008.

4. Agustín Rodríguez Chávez 2,010 Estructuras Flexibles De Costos Para Enfrentar La Globalización y La Competitividad En Las Empresas, Quipukamayoc Revista de la Facultad de Ciencias Contables Vol. 17 N.o 33 pp. 19-26 (2010) UNMSM, Lima, Perú. ISSN: 1560-9103 (versión impresa) / ISSN: 1609-8196 (versión electrónica)

5. Santiago Ibarra Mirón. http://www. monografias.com/trabajos16/configuraciones-productivas/configuracionesproductivas.shtml (visitado el 20-082011).

6. Antonio Romero- Administración y gerencia. http://www.gestiopolis.com/ recursos/documentos/fulldocs/ger/ outsourcingantonio.htm (visitado el 2208-2011). 